\title{
Design and assembly of double cross-over linear arrays of micrometer length using rolling circle replication
}

\author{
Daniel Lubrich, Jonathan Bath* and Andrew J. Turberfield
}

Department of Physics, University of Oxford, Clarendon Laboratory, Parks Road, Oxford OX1 3PU, UK.

*j.bath@physics.ox.ac.uk 


\begin{abstract}
We demonstrate the use of rolling circle replication to template linear DNA arrays whose sizes bridge the gap between nanometer-scale self-assembly and top-down lithographic fabrication. Using rolling circle replication we have produced an oligonucleotide containing several hundred repeats of a short sequence motif. On this template we have constructed, by self assembly, an array consisting of two parallel duplexes periodically linked by antiparallel Holliday junctions. We have observed arrays up to 10 micrometers in length by atomic force microscopy.
\end{abstract}

\title{
1. Introduction
}

A simple class of DNA nanostructure [1] is the one-dimensional DNA array [2-10]. One-dimensional arrays are suitable templates for the fabrication of DNA nanowires $[4,10,14,15,16]$ : they can have a longer persistence length than lambda DNA [3] and are expected to create a higher density of nucleation centres for metallization. DNA-templated wires have been used to connect molecular sized electronic components to lithographically defined electrodes [17]. There also is interest in using one-dimensional arrays to program the growth of two-dimensional DNA arrays $[5,6,18]$ and as tracks for linear DNA motors $[11,12,13]$.

Research into the design and assembly of one-dimensional arrays has focused on the traditional DNA tiling approach that has been employed successfully for two-dimensional array fabrication [19]. However, the cooperative stabilization that favours the growth of large two-dimensional arrays does not play a part in the assembly of simple one-dimensional chains of tiles. A theoretical model of onedimensional assembly [20] predicts that the concentrations of the assemblies formed are distributed according to $P_{x}=p^{x-1}(1-p)$, where $P_{x}$ is mole fraction of polymers containing $x$ monomers and $p$ is the extent of the reaction. Hence the monomeric "assembly" is always the most abundant. Recent experiments and computer simulations have confirmed this general result for one-dimensional arrays of DNA tiles [6]: the maximum array length observed was only a few hundred nanometers (about twenty 
tiles). An additional problem is that once a chain of tiles has grown to a length comparable to its persistence length there is a significant probability that its ends will join to form a ring, halting further growth [21]. Mean linear array lengths can be increased by increasing the stiffness of the structure [2]: tubular structures can grow up to tens of micrometers [8-10]. Cyclisation can also be avoided by using a non-equilibrium assembly strategy in which designed secondary structure makes monomers unreactive except to addition at the end of a growing chain [22].

Here we report a new approach to the near-equilibrium assembly of linear arrays that overcomes the length limitations that are inherent to simple tile-based approaches. An oligonucleotide containing several hundred copies of a designed base sequence is produced by rolling circle replication of a circular oligonucleotide [23-25]. An array assembled on this periodic template can extend up to the length of the template. We use atomic force microscopy (AFM) to compare one-dimensional arrays produced by this technique with arrays created by one-dimensional tile assembly.

\section{Results}

The nanostructure that we have designed in order to investigate templated array assembly is shown in Figure 1A; its structure is similar to that of a one-dimensional array of double-crossover (DX) tiles [26]. The topmost strand of DNA, indicated by a dotted blue line, is the continuous periodic template produced by rolling circle replication. Many copies of two oligonucleotides assemble on the template by hybridization of complementary sequences of bases to produce the array. The array consists of two extended, parallel double helices (which run horizontally in the figure). Every two helical turns (21 nucleotides) there is a Holliday junction (HJ) [27] at which two oligonucleotides cross between the helices, holding them together. A period of the array contains one HJ and is 21 nucleotides (nt) long.

The tile-assembly system that we have used for comparison is shown schematically in Figure $1 \mathrm{~B}$. The DX tile has the DAE structure [26] and is composed of five oligonucleotides which hybridize to create two parallel double helices; the tile is held together by two HJs. The DX tile has four single-stranded 
sticky ends; those on the right hand end of the tile are complementary to those on the left, so hybridization of sticky ends can join tiles together to form a one-dimensional array. The spacing between HJs, both within a tile and between adjacent tiles, is two helical turns (21 nt): the assembled array consists of two extended, parallel double helices linked by HJs every $21 \mathrm{nt}$. The period of the array (the length of a DX tile containing two inequivalent HJs) is $42 \mathrm{nt}$. Details of both structures, including the base sequences of all oligonucleotides, are given in the Supplementary Information; where possible the same nucleotide sequences were used in both.

Figure $1 \mathrm{C}$ illustrates stages in the production of the periodic strand to be used as a template for array assembly. Intermediate products are displayed in a gel in Figure 1D. The template for replication was a $63 \mathrm{nt}$ oligonucleotide containing three repeats of a $21 \mathrm{nt}$ motif (Figure 1D, lane 1). The 5 phosphorylated template was circularized with T4 DNA ligase using an excess of a 15 nt primer which acts as a splint to hold the $3^{\prime}$ and $5^{\prime}$ ends of the template together (Figure 1D, lane 2). Figure 1D lane 3 shows the circular ligation product that remains after all linear DNA was digested with Exonuclease I and Exonuclease III (New England Biolabs). Rolling circle replication reactions containing $\sim 10 \mathrm{nM}$ circular substrate, $50 \mathrm{nM}$ primer, $750 \mu \mathrm{M}$ of each nucleotide and 10 units of $\Phi 29$ polymerase (New England Biolabs) were incubated for $2 \mathrm{~h}$ at $30^{\circ} \mathrm{C}$ then stopped by phenol:chloroform extraction (Figure $1 \mathrm{D}$, lane 4). The $\Phi 29$ polymerase catalyses the replication of the circular template, starting from the $3^{\prime}$ hydroxyl group of the primer. The polymerase is capable of displacing the daughter strand ahead of itself as it moves around the circle many times: the product of rolling circle replication contains several hundred copies of a sequence complementary to the circular template.

Both arrays were assembled by mixing strands at a concentration of $2.5 \mu \mathrm{M}$ (estimated concentration of template $2 \mathrm{nM})$ in a hybridization buffer $(10 \mathrm{mM} \mathrm{MgCl}, 10 \mathrm{mM}$ Tris $\bullet \mathrm{HCl}, 1 \mathrm{mM}$ EDTA, pH 8.0) and cooling from $90^{\circ} \mathrm{C}$ to $25^{\circ} \mathrm{C}$ over $36 \mathrm{~h}$. Arrays were imaged by tapping-mode AFM (Veeco Nanoman) on freshly cleaved mica in air. Their height was measured to be between $0.8 \mathrm{~nm}$ and $0.9 \mathrm{~nm}$. The arrays produced by tile assembly are less than $250 \mathrm{~nm}$ in length; a typical image is shown in Figure 
2A. Individual tiles can be resolved: the periodicity of the tile arrays is in good agreement with the expected value of $14.3 \mathrm{~nm}$. Arrays built on periodic templates, shown in Figure 2B, C, E are significantly longer than tile arrays (compare Figure $2 \mathrm{~A}$ and $2 \mathrm{~B}$ ). The fine structure of the templated arrays is not resolved; this may be a result of their shorter period or of the lower density of nicks (where the $5^{\prime}$ and $3^{\prime}$ ends of adjacent oligonucleotides abut). The templated arrays are expected to have a persistence length similar to that of a DX tile array which has been measured to be $100 \mathrm{~nm}^{3}$. Many templated arrays are certainly much longer than their persistence length, making it impossible to obtain reliable length measurements from images such as Figure 2B, C. To measure the distribution of array lengths shown in Figure 2D, arrays were stretched out on silicon (Figure 2E). A $5 \times 5$ mm silicon wafer was cleaned in acetone and rinsed with water. A $20 \mu 1$ drop of hybridization buffer containing the linear arrays was then slowly pushed along the surface using a stream of nitrogen gas. The silicon was then again rinsed with water. Arrays up to $10 \mu \mathrm{m}$ in length were observed; Figure 2D shows the distribution of measured lengths.

The average concentration of binding sites on template strands was estimated to be an order of magnitude lower than the concentrations of the two oligonucleotides that assembled on them to produce the array. Under these conditions we expect all sites to be occupied and the lengths of the templated arrays to be equal to the lengths of the template strands. Our observed length distribution is consistent with the calculated distribution of lengths for the template if it is assumed that, during rolling circle replication, the probability per base added that the polymerase halts is $3 \times 10^{-4}$. Our mean array length, $3 \mathrm{~kb}$, is much shorter than anticipated given that the polymerase can replicate the entire $20 \mathrm{~kb} \Phi 29$ genome without dissociating from the template [25]; a reduction in the processivity of the enzyme when replicating a circle whose perimeter is less than the persistence length of double-stranded DNA is expected.

The use of rolling circle replication to create a periodic template makes it straightforward to place a unique label at one or both ends of the array. A unique sequence of bases or a chemical modification can 
be incorporated at the $5^{\prime}$ end of the template strand simply by extending the $5^{\prime}$ end of the primer (see Figure 1A). Chemical modification of the $3^{\prime}$ end of the template may be achieved by addition of an appropriately modified chain-terminating dideoxynucleotide during the course of polymerization.

\section{Conclusions}

The results presented here, along with two recent studies [28,29], demonstrate that rolling circle replication is a simple way to generate templates for nanostructures whose sizes bridge the gap between the nanometer scale of most molecular self-assembly and the micrometer scale of typical top-down fabrication. We believe that this technique has wide applicability in DNA nanofabrication, for example in creating structures that act as interfaces between self-assembled molecular devices and lithographically-defined electrodes.

Supporting Information Available. Details of DNA sequences and sequence design. 


\section{References}

[1] Seeman N C 2003 Nature 421427

[2] Yang X, Wenzler L A, Qi J, Li X, Seeman N C 1998 J. Am. Chem. Soc. 1209779

[3] Sa-Ardyen P, Vologodskii A V, Seeman N C 2003 Biophys. J. 843829

[4] Yan H, Park S H, Finkelstein G, Reif J H, LaBean T H 2003 Science 3011882

[5] Yan H, LaBean T H, Feng L P, Reif J H 2003 Proc. Natl. Acad. Sci. USA 1008103

[6] Schulman R, Lee S, Papadakis N, Winfree E 2004 LNCS 2943108

[7] Li H, Park S H, Reif J H, LaBean T H, Yan H 2004 J. Am. Chem. Soc. 126418

[8] Mitchell J C, Harris J R, Malo J, Bath J, Turberfield A J 2004 J. Am. Chem. Soc. 12616342

[9] Rothemund P W, Ekani-Nkodo A, Papadakis N, Kumar A, Fygenson D K, Winfree E 2004 J. Am. Chem. Soc. 12616344

[10] Liu D, Park S H, Reif J H, LaBean T H 2004 Proc. Natl. Acad. Sci. USA 101717

[11] Turberfield A J, Mitchell J C, Yurke B, Mills A P Jr, Blakey M I, Simmel F C 2003 Phys. Rev. Lett. 90118102

[12] Sherman,W B, Seeman N C 2004 Nanoletters 41203

[13] Shin J S, Pierce N A 2004 J. Am. Chem. Soc. 12610834

[14] Braun E, Eichen Y, Sivan U, Ben-Yoseph G 1999 Nature 391775

[15] Richter J, Mertig M, Pompe W, Monch I, Schackert H K 2001 Appl. Phys. Lett. 78536

[16] Keren K, Krueger M, Gilad R, Ben-Yoseph G, Sivan U, Braun E 2002 Science 29772 
[17] Keren K, Berman R S, Buchstab E, Sivan U, Braun E 2003 Science 3021380

[18] Rothemund P W K, Papadakis N, Winfree E 2004 PLOS Biology 22041

[19] Winfree E, Liu F R, Wenzler L A, Seeman N C 1998 Nature 394539

[20] Flory P J 1936 J. Am. Chem. Soc. 581877

[21] Jacobson H, Stockmeyer, W H 1950 J. Chem. Phys. 181600

[22] Dirks R M, Pierce N A 2004 Proc. Natl.Acad.Sci. USA 10115275

[23] Fire A, Xu S Q 1995 Proc. Natl. Acad. Sci. USA 924641

[24] Daubendiek S L, Ryan K, Kool E T 1995 J. Am.Chem. Soc. 1177818

[25] Blanco L, Bernard A, Lazardo J M, Martin G, Garmendia C, Salas M 1989 J. Biol. Chem. 264 8935

[26] Fu T J, Seeman N C 1993 Biochemistry 323211

[27] Holliday R 1964 Genet. Res. 5282

[28] Beyer S, Nickel P, Simmel FC 2005 Nanoletters 5719

[29] Deng Z, Tiang Y, Lee S-H, Ribbe A E, Mao C 2005 Angew. Chem. Int. Ed. 442 
A
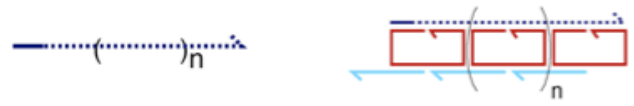

B
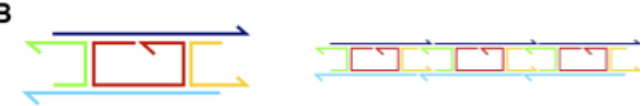

C

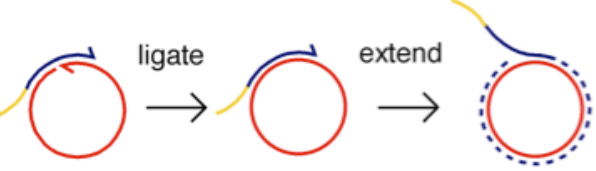

D

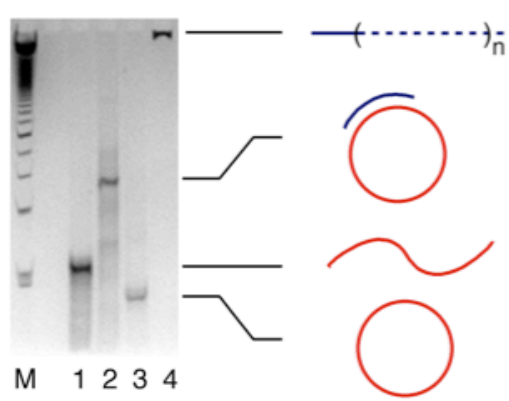

Figure 1. (A) The periodic template strand and linear array assembled on it. (B) Double-crossover tile and the linear array produced by tile assembly. (C) Production of a long, periodic template strand by rolling circle replication. The linear rolling circle substrate is closed by a splint and ligated to create a covalently closed circular strand. The splint acts as primer: its $3^{\prime}$ end is extended by a polymerase which replicates the circle many times, creating a periodic daughter strand. A non-complementary sequence at the $5^{\prime}$ end of the primer (yellow) may be used to create a unique label at the $5^{\prime}$ end of the daughter strand. (D) Rolling circle replication visualized on a silver-stained $12.5 \%$ native polyacrylamide gel. Lane 1: linear rolling circle template strand. Lane 2: splinted and ligated circle. Lane 3: as lane 2, after digestion of linear strands. Lane 4: products of the rolling circle replication. Marker: 50 base pair ladder (Amersham Pharmacia). 

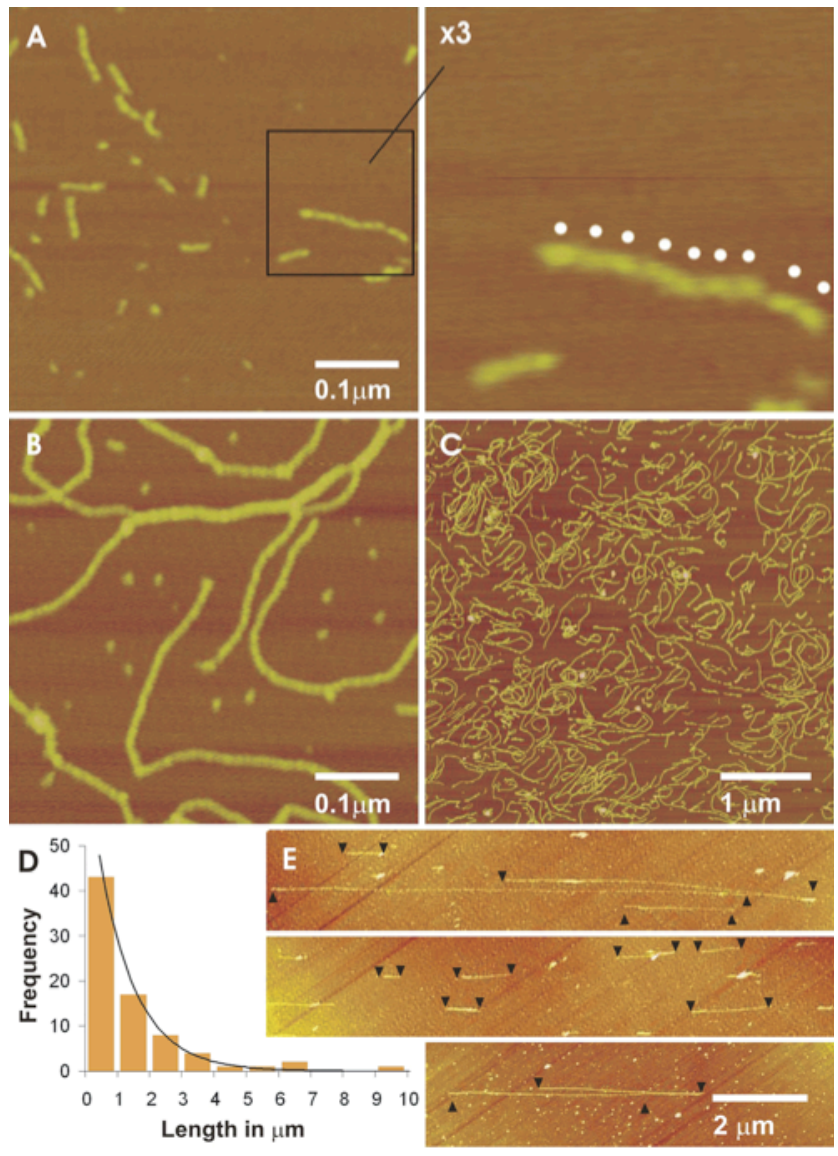

Figure 2. AFM images of tile arrays (A) and templated arrays (B,C,E) on mica. Measured array heights are between $0.8 \mathrm{~nm}$ and $0.9 \mathrm{~nm}$. (A) Image of tile arrays on mica. The boxed section is magnified in the right hand panel; individual tiles are indicated with white dots. $(B, C)$ Images of templated arrays on mica. (D) Distribution of lengths of templated arrays. The black curve shows the expected distribution of template strand lengths calculated as described in the text. The histogram shows the actual data, obtained from images as shown in (E). (E) Images of templated arrays stretched out on silicon. The ends of arrays are indicated. 